\title{
An Objective No-Reference Measure of Illumination Assessment
}

\author{
Gholamreza Anbarjafari \\ iCV Research Group, Institute of Technology, University of Tartu, Tartu 50411, Estonia, shb@ut.ee
}

\begin{abstract}
Illumination problems have been an important concern in many image processing applications. An important issue in the field of illumination enhancement is absence of a quantitative measure for assessment of illumination of an image. In this research work a quantitative measure indicating the illumination state, i.e. contrast level and brightness of an image is also proposed. The measure utilises the estimated Gaussian distribution of the input image and the Kullback-Leibler Divergence between the estimated Gaussian distribution and the desired Gaussian distribution to calculate the quantitative measure. The experimental results show the effectiveness and the reliability of proposed illumination assessment measure.
\end{abstract}

Keywords: Illumination assessment measure, quantitative image assessment, Kullback-Leibler divergence, image enhancement, measurement.

\section{INTRODUCTION}

$\mathrm{C}$ ONTRAST ENHANCEMENT is frequently referred to as one of the most important issues in image processing $[1,2,3,4,5]$. When analysing illumination methods, other than visual analysis evaluation (i.e. subjective evaluation), there is no objective quantitative method to assess the illumination of the enhanced images. Some researchers use the mean opinion scores (MOS) in order to compare different illumination techniques however MOS is a subjective metric. Other image quality metrics, like the Face Quality Index combine different properties (e.g. contrast, brightness, sharpness, illumination) of an image to achieve a metric used specifically in face recognition $[6,7]$. There is also a Universal Quality Index (UQI) that performs well if image distortion is being analysed $[8,9]$.

Another common approach is to use peak signal-to-noise ratio (PSNR) to assess the quality of an image. Using PSNR might be a good approach for a consistent, fixed content signal but when applied to images or videos it gives incomparable results across multiple contents $[10,11]$. Also it is important to note that in the case of illumination enhancement assessment the absence of the ground truth limits the use of some the aforementioned techniques. There exist measures that try to deal with no-reference (NR) quality measurement. These approaches use machine learning techniques such as sparse extreme learning machine and neural networks [12, 13]. They are based on the human visual system and try to emulate the MOS. In this paper we define a notation of an ideally illuminated image that will be the reference point for our metric. This does not fix one specific image but rather describes the properties that a mathematically ideal image should have.

We are proposing a new quantitative measure for image illumination quality which is based on finding the KullbackLeibler Divergence (KLD) between an estimated Gaussian distribution (GD) of a given image and the desired GD [14]. The desired GD can be chosen in a way that satisfies all the required and necessary features of an ideal enhanced image. The proposed metric generates a numerical value between -1 and 1 to reflect the quantitative assessment of illumination of a given image without using a ground truth image. A positive numeric value represents a high contrast image and the negative value corresponds to a low contrast image. If the numeric value is in $(-0.5,0.5)$ range then the image is a dark image otherwise it is a bright image. By using the proposed metric all four possible image illumination quality cases namely: low contrast-dark, low contrast-bright, high contrast-dark and high contrast-bright can be represented.

\section{Proposed image ILlUMination ASSESSMENT MEASURE}

Many quality measurement methods such as the PSNR and UQI calculations require a ground truth image, it would be useful to have a technique that did not have this requirement. This is the main motivation for introducing a measure which can be used to assess the illumination quality independent of the ground truth image. The proposed measure is based on statistical pixel intensity distributions of the given image and the so called ideal/desired image.

In this research work an image whose mean is 128 is considered to be an image with a good lightening. Therefore, we are considering that the GD of an image with desired illumination, has mean of $128(\mu=128)$ for an 8-bit grey scale image. The standard deviation $(\sigma)$ of the desired distribution should be calculated in order to estimate the GD of a desired image. It is clear that an 8-bit grey level image has the pixel range of $[0,255]$. Hence, the $\sigma$ should be calculated in such a way that the GD covers this range effectively. It is known that the following transformation is valid:

$$
X=G\left(\mu, \sigma^{2}\right) \stackrel{Z=\frac{x-\mu}{\sigma}}{\longrightarrow} Z=N(0,1)
$$

where $N$ is representing a standard normal distribution and $G$ is a GD. Assume that we want our estimated distribution to satisfy the following condition:

$$
P(0 \leq x \leq 255)=0.998
$$


Given that $\mu=128$, in order to find $\sigma$, one can easily follow the proceeding steps:

$$
\begin{array}{r}
P(0 \leq x \leq 255)=0.998 \\
P\left(-Z_{0} \leq z \leq Z_{0}\right)=0.998 \\
P\left(0 \leq z \leq Z_{0}\right)=\frac{0.998}{2}=0.499
\end{array}
$$

By using the table of standard normal curve areas we get $Z_{0}=3.09$ resulting in:

$$
Z_{0}=\frac{255-128}{\sigma} \rightarrow \sigma=\frac{255-128}{3.09} \approx 41.1003
$$

This calculation also holds for other probability values given in eqn. 4. Therefore a GD with mean 128 and standard deviation of $41.1003, G_{d}\left(\mu=128, \sigma^{2}=1689.2347\right)$, can be considered as a desired distribution. In addition for a given image the estimated GD of such image can be calculated. In order to precede it is important to note that the resulting GDs have been scaled in such a way that they have a peak value of 1 by using the following formula:

$$
G_{i m}\left(\mu_{i m}, \sigma_{i m}^{2}\right)(x)=\frac{G_{i m}\left(\mu_{i m}, \sigma_{i m}^{2}\right)(x)}{\max _{x}\left[G_{i m}\left(\mu_{i m}, \sigma_{i m}^{2}\right)(x)\right]}
$$

Now we can find the divergence between the estimated scaled GD of the input image, $G_{i m}$, and the desired scaled $\mathrm{GD}, G_{d}$ by using KLD. The lower this value, the better the illumination, because it represents how similar $G_{i m}$ to $G_{d}$ is. This value, $\kappa$, can be formulated as follows:

$$
\kappa\left(G_{i m}, G_{d}\right)=\sum_{j=0}^{255} G_{i m_{j}} \log \left(\frac{G_{i m_{j}}}{G_{d_{j}}}\right)
$$

where variable $j$ represents the possible grey level range which is $[0,255]$. Hence there is no need to include the $(-\infty, 1] \cup[256, \infty)$. In order to normalise $\kappa$, the divergence value should be divided by its maximum possible value. The maximum distance occurs when we have an impulse on one of the grey levels with the peak value equal to $\sum G_{d}$, e.g. $\sigma_{b}(\mathrm{j}=0)$ or $\sigma_{w}(\mathrm{j}=255)$. Let assume that this maximum KLD value is shown by $\kappa_{\max }$, which is as follows:

$$
\kappa_{\text {max }}=\sum_{j=0}^{255} \sigma_{w} \log \left(\frac{\sigma_{w}}{\sigma_{d_{j}}}\right)
$$

Therefore the normalized divergence can be calculated by:

$$
\bar{\kappa}\left(G_{i m}, G_{d}\right)=\frac{\kappa\left(G_{i m}, G_{d}\right)}{\kappa_{\max }}
$$

It holds that $\bar{\kappa} \in[0,1]$. When $\bar{\kappa}$ is equal to 0 , it means we have the perfect match between the $G_{i m}$ and $G_{d}$ scaled distributions, which means that input image has the ideal/desired illumination. Let?s define $\xi$ to be the illumination metric that would generate a similarity measurement of the estimated scaled $G_{d}$ of the input image and the desired scaled Gaussian distribution, which has a value between 0 and 1 . $\xi$ can be formulated by modifying eqn. 8 as follows:

$$
\begin{gathered}
\xi\left(G_{i m}, G_{d}\right)=\operatorname{sign}\left(\mu_{i m}-128\right) \frac{\bar{\kappa}}{2}+0.5 \\
=\operatorname{sign}\left(\mu_{i m}-128\right) \frac{\kappa\left(G_{i m}, G_{d}\right)}{2 \kappa_{\max }}+0.5
\end{gathered}
$$

where sign function is 1 for non-negative numbers and - 1 otherwise. Introducing sign function helps to differentiate the input image into dark and bright images. Image with $0 \leq \xi<$ 0.5 represents a dark image and image with $0.5<\xi \leq 1$ represents a bright image. In order to enrich the eqn. 9 so that the result can also give some idea about the contrast of the distribution of the image (i.e. the image has a low or high contrast) variance of the distribution will be taken into the account. Hence considering all these issues we modify the eqn. 9 and propose the following metric for illumination quality:

$$
\begin{array}{r}
\xi\left(G_{i m}, G_{d}\right)=\operatorname{sign}\left(\sigma_{i m}-41.1003\right)\left(\operatorname{sign}\left(\mu_{i m}-128\right)\right. \\
\left.\frac{\kappa\left(G_{i m}, G_{d}\right)}{2 \kappa_{\max }}+0.5\right)
\end{array}
$$

where $-1 \leq \xi \leq 1$ and $\xi_{\text {ideallyilluminatedimage }}=0.5$. In its final form, the sign of $\xi$ indicates the contrast of the image. If $\xi$ is a positive number it means that the image has a high contrast and if it is a negative number then it indicates that the image has a low contrast as shown in Fig. 1.

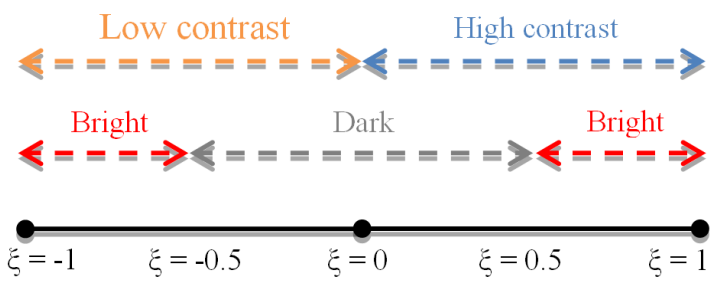

Fig. 1: $\xi$ guideline for determining illumination state

\section{EXPERIMENTAL RESULTS AND DISCUSSION}

Table 1 presents several images and their measure values for those, prior to and after applying singular value equalisation based illumination enhancement technique [4].

In order to verify the effectiveness of the proposed measure, also a mean opinion score (MOS) has been studied. MOS analysis has been done by asking 260 randomly selected students at University of Tartu and Eastern Mediterranean University to vote for 360 images with different illumination states namely, 90 dark images, 90 bright images, 90 low contrast images, and 90 high contrast images. The participants where provided by all the images in random order and they were asked to divide those images in the aforementioned categories. Table 2 shows the average results of MOS. Also 
Table 1: Experimental results prior to and after image enhancement
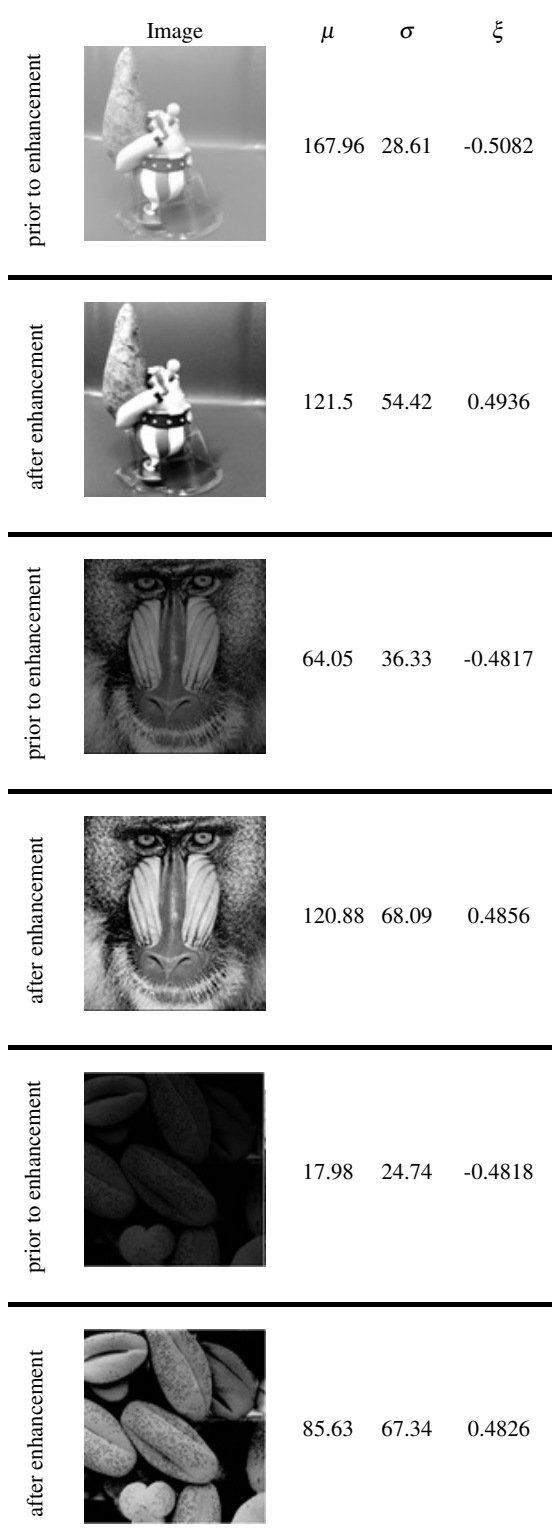

the $\xi$ value for each of these images have been calculated by using eqn. 10 and then the illumination state of the images have been assigned by using guideline shown in Fig. 1. Table 2 presents the results of MOS and proposed measure. Table 2 shows that the proposed measure is very effective in determining the illumination state of a given image. Some of the images used in the MOS analysis are shown in Fig. 2.

\section{CONCLUSION}

In this research work in order to overcome shortage of a quantitative measure for illumination assessment, a new measure was proposed. The quantitative results showed the proposed measure is a very good no-reference quantitative measure for illumination assessment.

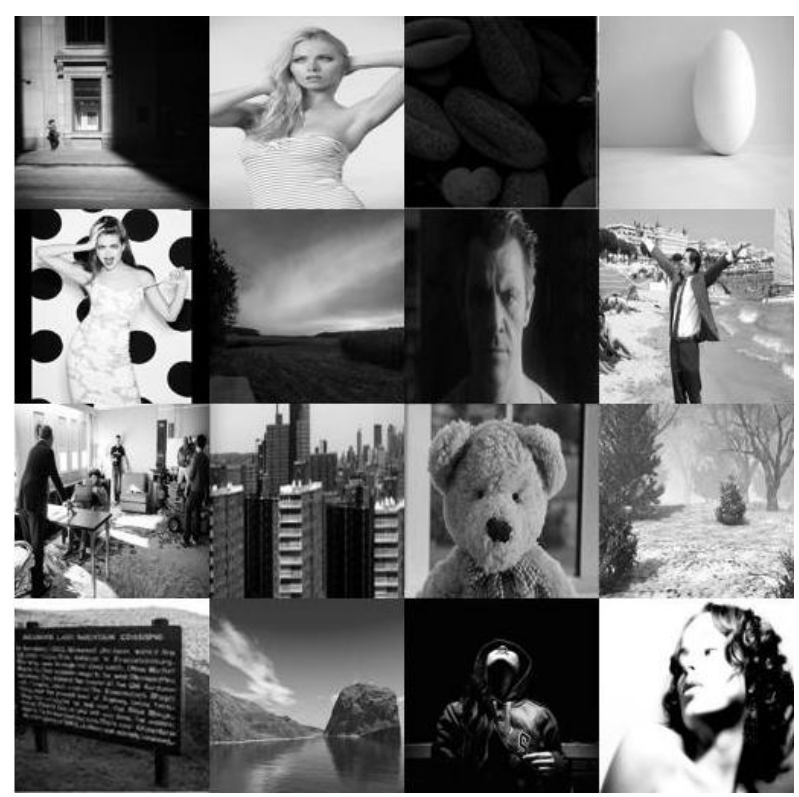

Fig. 2: Sample images used in MOS analysis

\section{ACKNOWLEDGEMENT}

The research was supported by the Estonian Research Council Grant (PUT638).

\section{REFERENCES}

[1] Du, S., Ward, R. K. (2010). Adaptive region-based image enhancement method for robust face recognition under variable illumination conditions. IEEE Transactions on Circuits and Systems for Video Technology, 20(9), 1165-1175.

[2] Anbarjafari, G. (2013). Face recognition using color local binary pattern from mutually independent color channels. EURASIP Journal on Image and Video Processing, 2013(1), art. no. 6.

[3] Sheet, D., Garud, H., Suveer, A., Mahadevappa, M., Chatterjee, J. (2010). Brightness preserving dynamic fuzzy histogram equalization. IEEE Transactions on Consumer Electronics, 56(4), 2475-2480.

[4] Demirel, H., Ozcinar, C., Anbarjafari, G. (2010). Satellite image contrast enhancement using discrete wavelet transform and singular value decomposition. IEEE Geoscience and Remote Sensing Letters, 7(2), 333-337.

[5] Hasikin, K., Isa, N. A. M. (2013). Fuzzy image enhancement for low contrast and non-uniform illumination images. In IEEE International Conference on Signal and Image Processing Applications (ICSIPA 2013), 8-10 October 2013. IEEE, 275-280.

[6] Abaza, A., Harrison, M. A., Bourlai, T. (2012). Quality metrics for practical face recognition. In 21st International Conference on Pattern Recognition (ICPR 2012), 11-15 November 2012. IEEE, 3103-3107. 
MEASUREMENT SCIENCE REVIEW, Volume 15, No. 6, 2015

Table 2: Illumination state assigned on an average to the images

\begin{tabular}{|c|l|c|c|c|c|}
\hline \multirow{2}{*}{$\begin{array}{c}\text { Illumination state } \\
\text { of the image }\end{array}$} & & \multicolumn{3}{|c|}{ Decision (\%) } \\
\cline { 2 - 5 } \multirow{2}{*}{ Bright } & decision based on average $\xi(\%)$ & 100 & 0 & & \\
\cline { 2 - 5 } & decision based on average MOS (\%) & 94.62 & 5.38 & & \\
\hline \multirow{2}{*}{ Dark } & decision based on average $\xi(\%)$ & 0 & 100 & & \\
\cline { 2 - 3 } & decision based on average MOS (\%) & 8.46 & 91.54 & & 0 \\
\hline \multirow{2}{*}{ Low Contrast } & decision based on average $\xi(\%)$ & & & 100 & 3.85 \\
\cline { 2 - 4 } & decision based on average MOS (\%) & & & 96.15 & 100 \\
\hline \multirow{2}{*}{ High Contrast } & decision based on average $\xi(\%)$ & & & 0 & 11.92 \\
\cline { 2 - 4 } & decision based on average MOS (\%) & & & 88.08 & Contrast \\
\hline
\end{tabular}

[7] Lin, W., Kuo, C.-C. J. (2011). Perceptual visual quality metrics: A survey. Journal of Visual Communication and Image Representation, 22(4), 297-312.

[8] Wang, Z., Bovik, A. C. (2002). A universal image quality index. IEEE Signal Processing Letters, 9(3), 81-84.

[9] Wang, Z., Bovik, A. C., Sheikh, H. R., Simoncelli, E. P. (2004). Image quality assessment: from error visibility to structural similarity. IEEE Transactions on Image Processing, 13(4), 600-612.

[10] Huynh-Thu, Q., Ghanbari, M. (2008). Scope of validity of psnr in image/video quality assessment. Electronics Letters, 44(13), 800-801.

[11] Sun, T., Zhu, X., Pan, J.-S., Wen, J., Meng, F. (2015). No-reference image quality assessment in spatial domain. In Genetic and Evolutionary Computing, Springer, 381-388.
[12] Ouni, S., Zagrouba, E., Chambah, M., Herbin, M. (2011). No-reference image semantic quality approach using neural network. In IEEE International Symposium on Signal Processing and Information Technology (ISSPIT 2011), 14-17 December 2011. IEEE, 106-113.

[13] Gaata, M., Sadkhn, S., Hasson, S. (2012). No-reference quality metric based on fuzzy neural network for subjective image watermarking evaluation. In IEEE International Conference on Imaging Systems and Techniques (IST 2012), 16-17 July 2012. IEEE, 315-320.

[14] Joyce, J. M. (2011). Kullback-Leibler divergence. In International Encyclopedia of Statistical Science. Springer, 720-722.

Received June 10, 2015. Accepted December 2, 2015. 